PART II

THE QUIET CHROMOSPHERE: DISK PHENOMENA 


\title{
WHY THE CHROMOSPHERE HAS ITS DISCRETE FINE STRUCTURE
}

\author{
HERMANN U. SCHMIDT \\ Max-Planck-Institut für Physik und Astrophysik, München, Germany
}

The fine structure in the chromosphere is probably controlled by magnetic flux concentrations which cellular convection produces in the photosphere. We will first deal with this flux concentration by the convection and its constraints. Then we will discuss the chromospheric network and its main constituent, the spicules. Following that we will look at the inside of the network, i.e. the fibrils.

\section{Convection and Magnetic Flux}

Cellular convection in supergranules and granules is well observed in the chromosphere and photosphere respectively. In these thin regions we know its sizes, $l$, velocities, $v$, and life times, $t$, as well as the mass density, $\varrho$. Therefore we can estimate the local dynamic stresses, $\varrho v^{2}$, which may counter-balance magnetic stresses, and a quantity with the dimension of a viscosity, $v l$, which may act on flows of larger scale as a turbulent viscosity or on the large scale magnetic flux as a diffusivity. But it is the ability of cellular convection to expel and to concentrate the magnetic field, which is of more importance for the understanding of our subject matter. More than $90 \%$ of the field is concentrated into small flux units at the border between adjacent supergranules (Frazier and Stenflo, 1972).

The transport of flux by cellular convection, its accumulation and its dissipation has been described by Parker (1963), Weiss (1966), and Clark and Johnson (1967). Clark and Johnston estimated the effect of pure horizontal transport at the top of an idealized hexagonal supergranular cell with reasonable extension and velocity. They found that if Lorentz forces would not interfer, an initially homogeneous vertical field at the top of this cell would in $14 \mathrm{~h}$ grow by a factor 55 at midboundary points, by a factor 400 at the vertices and decay to $6 \times 10^{-6}$ of its original value at the center. In reality the Lorentz forces stop this explosive accumulation much earlier since the dynamic pressure $\frac{1}{2} \varrho v^{2}$ in the photosphere would be balanced by the magnetic pressure $B^{2} / 8 \pi$ for a field of $50 \mathrm{G}$. But much larger fields, $>500 \mathrm{G}$, are observed. How can they be formed? There may be about 7 such units of $3 \times 10^{18} \mathrm{Mx}$ per supergranule, or about twice that number around its borderline. Therefore they are not too closely related to the less numerous vertices in the corners common to several supergranules. Instead their small size points to granules which happen to occur on the borderline and produce dynamic pressures equivalent to about $250 \mathrm{G}$ in the photosphere. The most thorough theoretical investigations relevant to this problem have been made by N. O. Weiss and his coworkers (e.g. Peckover and Weiss, 1972). They have computed the effects of a thermally driven two-dimensional cellular Boussinesq convection on 
the magnetic field with full account of the non-linear Lorentz forces. The most important result is that the simple concept of equipartition between kinetic and magnetic energy holds only under special circumstances. Instead their Figure 5 shows flux concentrations in which the magnetic energy is about ten times stronger than the total kinetic energy of the subdued convective motion whenever the effects of ohmic dissipation can be neglected against those of the Lorentz forces and for a wide range of parameters. The surplus of magnetic pressure over dynamic pressure is balanced by gas pressure. The magnetic field reaches a level (cf. their Figure 6) that suppresses the convection by balancing the differences of buoyancy in the temperature field, i.e. the magnetic pressure balances the full dynamic pressure of a cell without a field. Such effects are certainly operative at a level in the hydrogen convection zone where the supergranules are effective in transporting the solar heat flux. There the full dynamic pressure of the convection will balance magnetic pressures for $5000 \mathrm{G}$. Near the surface this effect seems to be inoperative, since the supergranulation has not been recognized in white light. However, it may be that the flux concentration can be localized in dark intergranular space by exact comparison. Therefore once more we must expect the dynamic pressure of the granules to be operative in the compression of the magnetic flux concentrations at the border of supergranules. It seems to be the combined action of supergranules and granules which produces these concentrations, within hours, at the borderline of a supergranule which confines new erupting flux e.g. in an arch filamentary system. Thereafter the flux concentrations forming a stretch of supergranular boundary may well live for a long time before they are dissipated, e.g. much longer than a single supergranule.

\section{The Ultraviolet Network}

The chromospheric network outlining the borders of supergranules is clearly recognized in $\mathrm{H} \alpha, \mathrm{Ca}^{+}$and in the UV up to a temperature of $1 \times 10^{6} \mathrm{~K}$ for the line formation (cf. Reeves and Parkinson, 1972). This was not expected, as Kopp and Kuperus (1968) predicted an anticorrelation between the Ca network and the UV emission pattern for the transition zone. Their Figure 1 indicated a weaker temperature gradient and thereby a stronger emission measure for those lines from the interior of the network cells. Here a major revision of this plausible picture is needed. An important step in this direction was done by Kopp (1972). He recognized another discrepancy which exists for the spherical symmetric model of the transition zone derived from the UV emission measures. These emission measures are inversely proportional to the temperature gradient at the temperature of line formation, so that a spherical symmetric temperature profile can be calculated. The conductive heat flow thus derived has a peak in divergence which would deposit more energy in the gas than what actually can be radiated at this temperature and density by a factor of about five, as can be seen from Kopp's Figure 2. It shows the calculated sink of the conductive flux and the source of the radiative flux. The sink peak is actually larger than the radiative losses by a factor of five for the appropriate pressure at the 
transition zone. Since mechanical wave energy sinks can only aggravate the problem, the conductive sink cannot be correct, but must be reduced by at least a factor of 5 . If this were to be an overall reduction of the temperature gradient, the observed emission measures could not be matched unless one assumes the observed radiation to originate from a network which covers on the average only one fifth of the visible Sun. If this is done the discrepancy is resolved and the total conductive flux of $6 \times 10^{5}$ erg $\mathrm{cm}^{-2} \mathrm{~s}^{-1}$ is reduced by a factor 25 to $2.5 \times 10^{4}$, since both the gradient and the area through which the conductive flux crosses back is reduced by a factor 5 . This is a drastic tentative change, but it is in line with all observations, especially of the continuation of the network into the corona. It implies that also the input of mechanical energy should be enhanced in the network or rather reduced in the interior of the supergranules. Such a relative enhancement of the mechanical input in the of the super-granules. Such a relative enhancernent of the mechanical input in the stronger magnetic field connected with the network is entirely reasonable, as the excitation of soundwaves is more effective under these conditions (cf. Parker, 1964). Over the interior of the supergranule we can assume that the field spreads out from the network very efficiently such that the vertical temperature gradient can be rather large. Also the density will be lower than in the network. Hence the total UV emission from the transition layer can be low, too, because the horizontal field insulates the overlying corona from the chromosphere against thermal contact even for a very large temperature gradient.

\section{Spicules}

We now turn to the most interesting and most puzzling inhabitants of the solar chromosphere, the spicules. They constitute the network in the chromosphere. The processes which have been considered to produce them are shockwaves guided by the enhanced field, thermal instability, diamagnetic accelerations, annihilation of opposite fluxes. In order to judge their applicability I will try to recall and to extrapolate some of the features of spicules reported by Beckers (1972). There seem to be about 80 spicules at any one time per supergranule, i.e. about an order of magnitude more than the number of underlying photospheric flux concentrations with which they certainly are correlated. They form groups or bushes around them, not simply a thin sheet, they do not follow all the directions of the field lines spreading out from the flux concentration but they seem to prefer the vertical direction slightly. This is certainly an extrapolation, as I assume that there must be a sizable amount of very low lying flux which insulates the cooler interior of the chromospheric supergranule from the corona, but does not guide spicules. The question whether the spicules can heat the corona has been answered negatively by Beckers because of the average upward flow of kinetic energy in the spicules is less than $10^{4} \mathrm{erg} \mathrm{cm}^{-2} \mathrm{~s}^{-1}$. Beckers also stresses the similarity of size, abundance and lifetime for granules and spicules. The spicules apparently rise with velocities near $20 \mathrm{~km} \mathrm{~s}^{-1}$, but may be less (cf. Grossmann-Doerth and von Uexküll, 1971). They reach nearly $10^{4} \mathrm{~km}$ with densities near 
$10^{11} \mathrm{~cm}^{-3}$ and temperatures near $15000 \mathrm{~K}$. Shockwaves as spicules have been proposed by Parker (1964), and Lüst and Scholer (1966) have done computations. But the results of these were not conclusive, as large amplitudes could not be reached. Since the material of the real spicule is rising with less than $25 \mathrm{~km} \mathrm{~s}^{-1}$ it cannot coast to the observed altitudes but only to less than $1 / 10$ of it if no external forces are applied to it during its rise. This simple argument of Pikelner (1971a) rules out many models in the literature.

Thermal instability in the chromosphere was first discussed by Athay and Thomas (1956) and by Kuperus and Athay (1967). The most thorough investigation of a linearized set of model equations for a pure one level hydrogen chromosphere was put forward by Defouw (1970a, b). This local instability is set up, whenever the total change of the net energy loss of a chromospheric plasma parcel with temperature under realistic conditions becomes negative. The most simple approach is to investigate this change statically under constant pressure. But also for more refined methods there seems to appear consistently near $17000 \mathrm{~K}$ an instability. With a positive temperature perturbation the increase of ionization diminishes the number of atoms that can cool the electrons in collisional ionizations and as the pressure nearly stays constant the number density itself must decrease so that the rate of cooling by collisions must decrease. The instability is especially sensitive in a magnetic field strong enough to align the motion in the perturbation. A quantitative discussion of the growth rates and the dependence on temperature, field and density looks quite good. But this does not mean too much, because once more after the onset of this instability the matter cannot rise very far unless its thermal velocity would reach $100 \mathrm{~km} \mathrm{~s}^{-1}$, which are not observed. It is also not clear whether the quiet chromosphere does not manage to hold the geometrical region of instability sufficiently small by a steep jump to higher temperatures, where other atoms can take over the cooling task. A small region which is only locally unstable may easily be stabilized by conduction and generation of waves at the boundary towards stable regions. There is also a model by Hollweg (1972). His Figure 7 shows supergranular flow towards the supergranular border which creeps downwards to avoid the magnetic field there. Above this flow spicules rise, also obeying alignment with the field. The balances of energy and momentum are not discussed. Instead, his Figure 8 illustrates the model in which mass conservation alone explains the spicule as the velocity $v$ moves in over $2 L \cdot z_{0}$ and the spicule moves out with $v_{\mathrm{s}}$ over $L \cdot d$. There is even an increase in $v_{\mathrm{s}}$ over that because the inward moving matter happens to obey a barometric formula which brings in a lot of mass at the bottom which has no choice but to move out at the top with the local density there:

$$
v_{\mathrm{s}}=v \cdot 2 \frac{h}{d}\left(e^{z_{0} / h}-1\right)
$$

which brings $40 \mathrm{~km} \mathrm{~s}^{-1}$ at $z_{0} \approx 1000 \mathrm{~km}$ out of $0.4 \mathrm{~km} \mathrm{~s}^{-1}$ at the bottom. In my opinion the model does not discuss the dynamics and therefore does not explain how a spicule overcomes gravity. 
Now we come to those models which involve external forces, which under the circumstances can be only magnetic, as the dynamic pressures above the photosphere are certainly insufficient. There are really two possibilities and they may be combined: dissipation or 'annihilation' of flux and/or diamagnetic acceleration. Schlüter (1957) put forward the latter process which is now often referred to as the 'melon seed mechanism'. It seems to me that this process probably is the only cause of the spicule. For the spicules, it was used, together with annihilation of opposite flux, by Pikelner $(1969,1971 \mathrm{a})$ and simultaneously by Uchida (1969). The diamagnetic process produces in an inhomogeneous magnetic field a net volume force on an imbedded body which is field free or has its own disconnected field. This force is proportional to $\nabla B^{2}$ and its strength depends on the surface and will change as the diamagnetic body expands in the process. Livshits and Pikelner (1964) have done calculations for ejecta from active regions, readily applicable to surges. The process basically does not run at the expense of magnetic energy but of the internal energy of the diamagnetic body. By its pressure it must push on the field, which is restored after the body has moved past. Therefore this is an ideal mechanism for recurrent phenomena. It may also act on bodies, which after an acceleration and some cooling in the expanded phase in a weaker field fall back by gravity, get new heat in the compressed phase, e.g., by radiation and then start on the same path again. The problem is always to store sufficient internal energy in the body and to get it disconnected from its magnetic environment. Therefore both authors quite naturally tried to use dissipation of magnetic flux as a source of internal energy. The statement that at the supergranular boundaries opposite polarities are mixed was once fashionable but it certainly is not nowadays. However, this may change again with better resolution. In any case, once you have opposite polarities you can easily get reconnection of field lines by Petschek's mechanism and you also get the needed increase in internal energy more easily (see Figure 1 of Pikelner). But this reconnection may be rather unimportant for the spicules, because there is probably not sufficient flux available in the solar cycle to supply all the spicules with their share. My estimate of the average production of surface flux by the solar dynamo is $10^{16.9} \mathrm{Mx} \mathrm{s}^{-1}$ and for the solar wind losses I get with a lifetime of 3 rotations $10^{16.3} \mathrm{Mx} \mathrm{s}^{-1}$. On the other hand for the spicules I get a conservative estimate of $10^{18.9} \mathrm{Mx} \mathrm{s}^{-1}$. Therefore $\mathrm{I}$ am afraid, we have to look for deeper sources of energy than reconnection. Certainly the dynamo production has to be reconnected too, but we may simply say that this phenomenon should be harder to find for the eager observer, as it is probably hidden among more than a hundred spicules. We do not know yet where the spicules get there heat. But we should remember Beckers statement, that we do not need very much, some $10^{3} \mathrm{erg} \mathrm{cm}^{2} \mathrm{~s}^{-1}$. My tip would be the same as the one of Beckers, i.e. to look into the layers underneath. There is a downstream flow towards the supergranule proper in the hydrogen convection zone. With the granulation this downstream pattern changes a bit to and forming a new azimuth with respect to an irregular field concentrations. Thereby, diamagnetic matter gets trapped near to the photosphere and in the field, is heated 
up and shot out. Anyway, it seems conceivable that observers may soon find a nice new 'moving diamagnetic body' which produces the spicule.

\section{Inside the Network}

Here we find the fibrils. One might ask though: do fibrils appear in the quiet chromosphere? I did not check whether there is a clean-cut answer to this question. In any case I shall discuss them briefly. They seem to outline a roughly continuous field of two-dimensional streamlines which apparently is confined to the chromosphere and mostly to parts of the active chromosphere. I do not now discuss the question whether this is the magnetic field, I simply assume it. They are probably confined to the active chromosphere for a simple reason, which is not so much the amount and the concentration of the flux but rather the fact that in the quiet chromosphere there is a much smaller chance to find a field line which does not leave the particular supergranular cell and therefore the chromosphere and even the corona for interplanetary space. Now why do we see this field? Meyer and Schmidt (1968a, b) argued that low lying fluxtubes connect opposite magnetic polarities of slightly different fieldstrength and, therefore, slightly different pressure. The high pressure will cause subsonic upword flow to the crest and supersonic downward flow which is dissipated in a shock into a subsonic inflow toward low pressure. These differences in density and pressure may become visible as contrast between fibrils.

Pikelner (1971b), on the other hand, has constructed a good quantitative model for fibrils, which assumes that they constitute the middle chromosphere between 1500 and $4000 \mathrm{~km}$, but that they are imbedded into a hot corona. This is possible because the plasma tube aligned by the magnetic field of several Gauss is almost perfectly insulated against heat conduction from the corona. Further it is assumed that the maximum height is limited because at this height the chromospheric thermal instability limit of $18000 \mathrm{~K}$ determined by Defouw is reached. The pressure distribution in the fibrils has to be hydrostatic, at least in its subsonic parts. In its supersonic parts the pressure will be even lower. Now the roughly known temperature profile in the lower chromosphere with fixed points $6000 \mathrm{~K}$ at $1000 \mathrm{~km}$ and $10000 \mathrm{~K}$ at $2000 \mathrm{~km}$ can be extrapolated for the fibrils so that at their visible crest at $4000 \mathrm{~km}$ the stability limit of Defouw mentioned above is reached, i.e. $18000 \mathrm{~K}$. Now the density can be determined from hydrostatic equilibrium and one fixed value, say $8 \times 10^{10} \mathrm{~cm}^{-3}$ at $2000 \mathrm{~km}$. Once that is known the ionization of hydrogen and the optical depth of the fibril in $\mathrm{H} \alpha$ at each height can be calculated from a detailed non-LTE model. It is found to be larger than 0.25 for the temperature range from 13000 to $18000 \mathrm{~K}$ in contrast to the transparent corona. Thus an $\mathrm{H} \alpha$ filtergram should show the fibril structure as it does.

Quite naturally the optical depth in $\mathrm{H} \alpha$ drops rapidly at the upper edge for the same reason that causes the thermal instability. The gas pressure at this level in the fibril is below the pressure of the ambient surrounding corona by a factor 5 . This 
does not cause a difficulty for the model as the magnetic field in the fibril has to compensate the total coronal pressure.

\section{Bright Points}

I want to add a few words about the bright points in the wings of $\mathrm{H} \alpha$ as they are also an important fine struçture: For a shortlived very local heating in a strong magnetic field concentration in the lowest chromosphere one would expect the same characteristics for three rather different processes, which all can occur under proper circumstances. They are ohmic dissipation of magnetic energy, viscous dissipation of kinetic energy and diamagnetic buoyancy of matter heated beneath the photosphere. In the first case the field concentration contains opposite fluxes and as it is formed it is annihilated. This would be the process for spicule formation described by Pikelner and Uchida, but which will probably form only one spicule out of many. It would be identified by resolution of the flux polarities as they move together. In the second case matter falls back down from sufficient height into the flux concentration so that its energy produces sufficient dynamic pressure to balance the gas pressure in the lower chromosphere or more. In the third case the internal energy is provided to a diamagnetic body below the chromosphere, so that buoyancy brings it up to the chromosphere.

In conclusion I list some open questions.

(1) From the numerical studies of the Cambridge group we have seen that in thermally driven cellular convection the enhancement in the magnetic energy can surpass the kinetic energy in the cell by factors up to 10 . The local magnetic pressure may then still be larger compared to the actual dynamic stresses, since it is balanced in this case by pressure gradients. Here is certainly a new good tool invented and applied by $\mathrm{N}$. O. Weiss and his coworkers which will in the future tell us more about the effects of solar surface convection on the magnetic field. The questions one might ask here are:

Can one measure the pressure gradients in the photosphere which balance the magnetic pressure of the field concentrations?

What are the exact phenomenological correlations between the field concentrations and the granules? e.g. do the concentrations fall into intergranular regions, is there a measurable low in the pressure and in the temperature? How do the granules apply their dynamical pressure?

(2) What are the correlations between granules and supergranules? Do the granules move towards supergranular boundaries or vertices? Is the chromospheric supergranule driven locally by thermal gradients or by some viscous coupling to deeper layers? Simon (1967) answered the second question positively. This thorough and labourious work was never repeated, but it concerns a fundamental question.

(3) What is the exact variation of the input in mechanical energy with the vertical magnetic field in the photosphere and with height in the chromosphere? We may infer from Parker's discussion of the influence of the magnetic field on the production 
of acoustic energy and from the XUV observations and their discussion by Kopp that the input is concentrated in the field underlying the network.

(4) What is the height, the thickness, and the pressure of the transition zone as the field varies? One might tentatively answer these questions in the quiet chromosphere using the assumption that the mechanical energy moves straight up. i.e., exactly radially outward, whereas the conduction follows a force free field configuration and hydrostatic equilibrium holds along those field lines. There is a danger that due to the widespread fear to make any inferences about the magnetic field we do not get out the fantastic amount of information contained in the basic observations. Important contributions to this symposion concerning these questions have been made by Jordan (1974) and by Gabriel (1974).

(5) What is the cause of the spicule? Is it diamagnetic acceleration driven by internal energy with input from annihilation of magnetic flux or by mechanical heating? The latter may occur during the time between the rise of repetitive spicules at the same stable location. What are the correlations between spicules and granules? Is magnetic annihilation ruled out as a cause by unipolarity on the small scale?

(6) Are the fibrils imbedded into the corona and down to which level is this possible? Being horizontal are the fibrils magnetically aligned? Is there an observable discontinuity of the magnetic field and its direction somewhere between the fibrils and the spicules?

\section{References}

Athay, R. G. and Thomas, R. N.: 1965, Astrophys. J. 123, 299.

Beckers, J. M.: 1972, Ann. Rev. Astron. Astrophys. 10, 73.

Burton, W. M., Jordan, C., Ridgeley, A., and Wilson, R.: 1974, this volume p. 89.

Clark Jr., A. and Johnson, A. C.: 1967, Solar Phys. 2, 432.

Defouw, R. J.: 1970a, Astrophys. J. 160, 659, 161, 55.

Defouw, R. J.: 1970b, Solar Phys. 14, 42.

Frazier, E. N. and Stenflo, J. O.: 1972, Solar Phys. 27, 330.

Gabriel, N. H.: 1974, this volume, p. 295.

Grossmann-Doerth, U. and von Uexküll, M.: 1971, Solar Phys. 20, 31.

Hollweg, J. V.: 1972, Cosmic Electrodyn. 2, 423.

Kopp, R. A.: 1972, Solar Phys. 27, 373.

Kopp, R. A. and Kuperus, M.: 1968, Solar Phys. 4, 212.

Kuperus, M. and Athay, R. G.: 1967, Solar Phys. 3/4, 361.

Livshits, M. A. and Pikelner, S. B.: 1964, Astron. Zh. 41, 464.

Lüst, R. and Scholer, M.: 1966, Z. Naturf. 21a, 1098.

Meyer, F. and Schmidt, H. U.: 1968a, Z. angew. Math. Mech. 48, 218.

Meyer, F. and Schmidt, H. U.: 1968b, Astron. J. 73, 72.

Parker, E. N.: 1963, Astrophys. J. 138, 226, 552.

Parker, E. N.: 1964, Astrophys. J. 140, 1170.

Peckover, R. S. and Weiss, N. O.: 1972, Comp. Phys. Commun. 4, 339.

Pikelner, S. B.: 1969, Astron. Zh. 46, 328.

Pikelner, S. B.: 1971a, Commun. Astrophys. Space Phys. 3, 33.

Pikelner, S. B.: 1971b, Solar Phys. 20, 286.

Reeves, E. M. and Parkinson, W. H.: 1972, Solar Phys. 24, 113.

Schlüter, A.: 1957, in H. C. van de Hulst (ed.), 'Radio Astronomy', IAU Symp. 4, 356.

Simon, G. W.: 1967, Proc. Capri Sympos. Chromospheric Fine Structure.

Uchida, Y.: 1969, Publ. Astron. Soc. Japan 21, 128.

Weiss, N. O.: 1966, Proc. Roy. Soc. A293, 310. 


\section{DISCUSSION}

Pecker first raised the question of the relationship between granulation and magnetic fields. He referred to a study initiated by his group in which some correlation appeared to exist between granules and magnetic fields measured on a granula scale. Kiepenheuer reported that, in well-resolved $\mathrm{H} \alpha$ line wing filtergrams showing mottles and granulation, there are cases where the foot points of the mottle (or spicule) seem to be well correlated with the intergranular region. Deubner emphasized the importance of sequential series of spectra in different spectral regions covering the whole extent of the atmosphere. He mentioned that some observations of this kind Also point to a close relationship between spicules and granules. Grossmann-Doerth pointed out that, if fibrils are the elongated small features which stretch out over distances at least as far as the diameter of supergranular cells, they do not appear in the quiet chromosphere but only in the active chromosphere. He then went on:

Grossmann-Doerth: May I ask a question concerning the driving mechanism of spicules: do you mean to say that Pikel'ner has shown that no thermal instability can be the cause of spicules? Did I understand this correctly?

Schmidt: Yes, Pikel'ner argues that you have to supply sufficient energy to overcome the difference in gravitational potential. Now, you can take any model, even constant temperature gradient through the unstable layer and if you now accelerate an element from the bottom to the top of this unstable layer by this thermal instability, you would get only a few kilometers per second. Thus you may reach an elevation of $10 \mathrm{~km}$ or so.

Sturrock: It is my impression that spicules and surges represent the same phenomenon occurring on different scales. In each case, a mass of cool gas is shot up; sometimes it disperses and sometimes it falls back to the surface. No observer has pointed out to me any way of distinguishing between spicules and surges (other than length and time scales). If there is indeed a common mechanism for driving spicules and surges, this points towards a magnetic model such as Schluter's or Pikel'ner's, and probably rules out a radiation-driven model such as Athay's.

Schmidt: I certainly agree with you, and I put the very same question to Le Roy and Rust when they were working on surges and they agreed. There is a paper by Lifshits and Pikel'ner (Soviet Astron. AJ 8 (1964), 368) which describes the diamagnetic acceleration of surges with surprising quantitative detail. The same description may be adequate for spicules when scaled down.

Most of the remaining discussion was concerned with the interaction between magnetic and velocity fields in the Sun. Because of its basic importance it is reported here in some detail. The question was first raised by:

Stix: You mentioned that in the photosphere the supergranulation has not enough kinetic energy to produce flux densities of $1000 \mathrm{G}$ but that the granulation possibly has enough. How can the granulation concentrate flux on a much larger scale than its own? Do you mean flux transport by turbulent diffusion?

Schmidt: I don't think so. The supergranulation cannot concentrate the flux up to $1000 \mathrm{G}$, but it can transport it. Weiss and Peckover have shown that cellular convection can concentrate the flux to a field strength above the equipartition value but for supergranules this would only give field strengths of order $60 \mathrm{G}$. The granules, on the other hand, cannot transport flux very effectively but they can concentrate it, probably up to field intensities of $1000 \mathrm{G}$. Later Meyer returned to the problem:

Meyer: According to computations by Weiss, which were carried out using the Bousinesq approximation, one gets a pressure decrease in the concentrated magnetic field region. In the non-Bousinesq case one must also take the vertical temperature structure into account and it may be that the reduced gas pressure produced by the supergranules in the lower regions also occurs at higher levels. If so, this pressure difference may assist in concentrating the magnetic fields.

Schmidt: I think you are right. In two recent papers Parker has argued that the granules impose a depression on the flux tube by two processes: turbulent pumping producing downward flow and the Bernoulli effect. I do not know whether we need these processes. The model calculations of Weiss and Peckover point in a somewhat different direction. The influence of the magnetic field on the thermal diffusion may well be such that the temperature depression does not spread out away from the magnetic column.

Vrabec: My comment is in response to that of Dr Stix. We generally believe that supergranules are responsible for sweeping any fields occurring in the cell interiors out to the cell boundaries, where the fields become concentrated into the supergranular network. Stix has expressed concern, because the supergranules cannot produce the field strengths of $1500 \mathrm{G}$ or more, presently believed to occur in this network. Let me point out that Dr Schmidt has just told us that normal granulation, on the other hand, can produce these high field strengths. Can we then not think of normal granulation producing and maintaining the 
high, localized fields, and supergranulation carrying the granulation and the field concentrations continually being produced by it, out to the cell boundary? The field structure is then the result of two processes acting independently. Granulation produces the high field strengths and fine structure, while supergranulation arranges these fields into the familiar network.

An important distinction between model calculations and the solar problem was pointed out by Gabriel.

Gabriel: Models such as you describe for the time taken for a convection cell to sweep the field to its edges are all based upon the starting point of a uniform field distribution. This does not occur in reality. It is more realistic to consider the starting point as the field concentrations remaining from the old decaying network. I suggest that this picture may significantly shorten the time required to set up the new field concentrations.

Schmidt: Yes, I agree. This general description of the changes in the supergranule network is supported by the Aerospace movie taken at Thule.

These views regarding the roles played by granule and supergranule velocity fields in producing the observed network fields appeared to gain some degree of general support in this and other discussions during the Symposium. However, Zirin added a word of caution.

Zirin: I am still worried about the business of convection concentrating fields, for several reasons. The observations themselves are extremely subtle, mostly based on the work of Leighton and his co-authors years ago and on this rather subtle work of Simon. I am impressed myself with the possibility from the limited amount of white-light films that we have that granules in fact represent an oscillation with a 5-min period rather like the calcium network and not convection. Furthermore, even if the flow is observed there it is possible that this is simply part of the spicule outflow being supplied from the centre of the network and not necessarily convection. We know that the network itself is a very stable feature and we would expect stronger convection there to balance this magnetic pressure. If this were not so, the stronger the field the less long it would live. So, I'm afraid we've built a house of cards on not a very strong foundation.

Schmidt: Well, I think the foundation is not too bad because we can base it on numerical simulations of stationary convection. I placed the migration of granules on my list only as a very important question in its own right. But I think it's important to know whether the supergranulation is moving the photosphere or not. The concentration of flux by the granules is effective everywhere because they cover the whole photosphere. They do not have to move. They simply expel the flux and provides sufficient buoyancy stresses to balance the field up to $1000 \mathrm{G}$.

Zirin: Would the convection have to be stronger where the field is stronger?

Schmidt: No. It may actually be weaker. This prediction of these calculations would be very interesting to test. As you saw in the figure, the convective velocity goes slightly down when the field strength goes up. Nevertheless, the field energy can surpass even the level of the total kinetic energy calculated for the undisturbed cell without any magnetic field.

Wilson: I think Zirin's question also raises the very important point that the surface velocity fields which we call the supergranulation are only the surface effects of velocity fields which, if they are convective in origin, must be most efficient at depths of order $10000 \mathrm{~km}$. We know this because attempts to find temperature correlations with the velocity fields have failed significantly. This then supports the picture that the deep supergranule velocity fields are quite adequate to transport magnetic flux to the cell boundaries. While we seem to agree that the granules cannot transport fields, the assertion that they can concentrate fields into small flux units of strengths $\sim 1000 \mathrm{G}$ is based at present on the numerical simulations of Weiss and Peckover and to a lesser extent on arguments presented here by Meyer and by Schmidt. It will be particularly important to further probe this question for if the overall picture as outlined in this discussion can be confirmed, it represents an important advance in our understanding of the relation between solar velocity and magnetic fields. 